\title{
Calidad seminal e inseminación intrauterina: estudio retrospectivo
}

\section{Semen Quality and Intrauterine Insemination: A Retrospective Study}

\author{
Luisa F. Calderón-Mendoza ${ }^{1,2}$ Lucero Castrillón López ${ }^{1} \quad$ Carlos Felipe Vélez Giraldo ${ }^{1}$ \\ Walter D. Cardona-Maya ${ }^{3}$ Verónica Isaza Álvarez ${ }^{1}$ \\ ${ }^{1}$ Centro de Medicina Reproductiva, Concevidas, Medellín, Colombia \\ 2 Estudiante de Maestría, Universidad CES, Medellín, Colombia \\ ${ }^{3}$ Grupo de Reproducción, Departamento de Microbiología y \\ Parasitología, Facultad de Medicina, Universidad de Antioquia, \\ Medellín, Colombia

\begin{abstract}
Address for correspondence Walter D. Cardona-Maya, PhD, Grupo de Reproducción, Departamento de Microbiología y Parasitología, Facultad de Medicina, Universidad de Antioquia, Carrera 53 \# 61-30, Sede de Investigación Universitaria, SIU; Laboratorio 534, Medellín, Antioquia, Colombia (e-mail: wdario.cardona@udea.edu.co).
\end{abstract}

Urol Colomb 2018;27:266-271.

\section{Resumen \\ Palabras Claves \\ - espermatozoide \\ - inseminación intrauterina \\ - embarazo \\ - fertilidad \\ - factor masculino}

\section{Abstract}

Objetivo El objetivo de este estudio fue evaluar los parámetros seminales en muestras seminales frescas y seleccionadas y determinar los factores que predicen el éxito de la inseminación intrauterina (IIU).

Materiales y Métodos Todas las parejas que se sometieron a IIU se registraron retrospectivamente y se incluyeron un total de 419 ciclos. Las muestras de semen fueron seleccionadas usando el método swim-up.

Resultados No se encontró un parámetro seminal que pueda ser considerado como predictor de embarazo. Después de la preparación de la muestra, hubo una mejoría en la movilidad espermática y una disminución en la concentración. Adicionalmente, una diferencia significativa en el volumen entre los individuos menores de 40 años y mayores de 40 años fue observada.

Conclusión No existen parámetros seminales que puedan predecir el éxito de la inseminación, básicamente debido a que lograr un embarazo depende de muchos factores. Sin embargo, el procesamiento en el laboratorio mejora considerablemente la movilidad espermática de las muestras y la IIU tiene tasas de embarazo aceptables; por lo tanto, debe ser considerada como primera elección de tratamiento.

Objective The aim of the study was to evaluate the sperm parameters in fresh and washed samples and define the factors for predicting success after intrauterine insemination (IUI).

Materials and Methods All couples undergoing IUI were retrospectively enrolled, a total of $419 \mathrm{IUI}$ cycles were included. Sperm samples were prepared using the swim-up. received

April 21, 2017

accepted

July 19, 2017

published online

May 25, 2018
DOI https://doi.org/

$10.1055 / \mathrm{s}-0038-1656517$.

ISSN 0120-789X.

eISSN 2027-0119.
Copyright (c) 2018, Sociedad Colombiana License terms de Urología. Publicado por Thieme Revinter Publicações Ltda., Rio de Janeiro, Brazil. Todos los derechos reservados. 

Keywords
- sperm
- intrauterine insemination
- pregnancy
- fertility
- male factor

Results There were no seminal parameters that could be considered as being predictors of pregnancy. After sperm washed, there was an improvement in sperm motility and a decrese in sperm concentration. There was also a significant difference in volume between individuals under 40 and those over 40 .

Conclusion There are no seminal parameters that can predict the success of the insemination, basically because achieving a pregnancy is due to many factors. However, laboratory processing considerably improves the sperm motility of samples and IUI has acceptable pregnancy rates; therefore it should be considered a first choice treatment.

\section{Introducción}

La inseminación intrauterina (IIU), es un tratamiento de reproducción asistida que se puede utilizar para contrarrestar la infertilidad en ciertos grupos de pacientes, ${ }^{1}$ consiste en depositar los espermatozoides previamente seleccionados en la cavidad uterina a mujeres que han recibido una estimulación ovárica. ${ }^{2}$ Tiene una variedad de indicaciones entre las que se incluye el factor masculino leve, hostilidad cervical e infertilidad de origen desconocido. $^{3}$ La IIU se considera como una técnica de reproducción asistida simple y de bajo costo ${ }^{4}$ e incluso gracias a su eficacia actualmente se reconoce como un tratamiento de reproducción asistida de primera línea para la mayoría de parejas infértiles, ${ }^{1}$ debido a que alcanza tasas acumulativas de embarazo similares a las obtenidas en un solo ciclo de técnicas de reproducción asistida más complejas. $^{2}$ Obtener entre el $10 \%$ y el $20 \%$ de embarazos clínicos por ciclo es un rango aceptable para la IIU. ${ }^{5}$

Muchos factores reportados han sido utilizados como predictores de éxito después de la IIU: la edad, ${ }^{6}$ la estimulación ovárica, ${ }^{7}$ la reserva ovárica de las mujeres ${ }^{8}$ y los parámetros espermáticos. ${ }^{4}$ Varios parámetros seminales y espermáticos se han correlacionado con el embarazo clínico después de la IIU incluyendo el número de espermatozoides móviles y el porcentaje de espermatozoides con morfología normal. ${ }^{3}$ Sin embargo, no existe un consenso sobre qué parámetros seminales puedan predecir el logro de un embarazo después de realizar una IIU y aún existe controversia con respecto a la predicción del embarazo teniendo en cuenta los parámetros espermáticos.

Por lo tanto, el objetivo del presente estudio retrospectivo fue evaluar los parámetros espermáticos en muestras frescas y seleccionadas y definir los factores que puedan predecir el éxito de la IIU.

\section{Materiales y Métodos}

Todas las parejas sometidas a la IIU que asistieron al Centro de Medicina Reproductiva ConceVidas (http://www. concevidas.com), fueron registradas retrospectivamente. Se incluyeron un total de 419 ciclos de IIU entre 2012 y 2016. En el estudio, sólo se incluyeron los casos de pacientes con muestras de semen frescas y se excluyeron los casos en los cuales se usó semen congelado o muestras de semen de donante. Se consideraron como parejas elegibles, todas en las cuales la mujer tenía al menos, una trompa de Falopio permeable y una cavidad endometrial normal. Así mismo, se incluyeron parejas con infertilidad por factor masculino, siempre y cuando se observaran espermatozoides móviles en el eyaculado. Se incluyeron parejas que han intentado lograr un embarazo por un período de un año o más; parejas con infertilidad masculina leve o moderada con un recuento de espermatozoides móviles mayor a 3 millones post-selección. Finalmente, se incluyeron parejas diagnosticadas con factor cervical, endometriosis, incapacidad para depositar el semen en la vagina e infertilidad de origen desconocido.

Las muestras de semen que tuvieron una concentración espermática $\left(\geq 15 \times 10^{6}\right.$ espermatozoides $\left./ \mathrm{mL}\right)$, movilidad progresiva $(\geq 32 \%)$ y morfología espermática $(\geq 4 \%)$ se consideraron como normales, de acuerdo a los criterios de la Organización Mundial de la Salud (OMS) 2010. ${ }^{9}$

Todas las muestras de semen fueron recibidas en el laboratorio después de 3 a 6 días de abstinencia sexual. Se evaluaron parámetros macroscópicos (volumen, pH, apariencia, viscosidad) y microscópicos (concentración espermática, viabilidad y movilidad) después de la licuefacción. Además, se prepararon extendidos del eyaculado para analizar la morfología siguiendo los criterios de la OMS. ${ }^{9}$ Así mismo, se calculó la movilidad total y el recuento total de espermatozoides móviles en muestras frescas y seleccionadas.

La inducción de la ovulación se realizó de acuerdo a la situación particular de cada mujer. En general, se utilizaron dos esquemas: a) Citrato de clomifeno (Zimaquin, Gynopharm, Bogotá, Colombia) y hormona folículo - estimulante urinaria (FSH) (Gonagam-hMG, Zone Pharma, Baesweiler, Alemania) o Menopur ${ }^{\circledR}$ (Ferring Pharmaceuticals; Saint-Prex, Suiza); o b) FSH recombinante (GONAL-f®, Merck, Darmstadt, Alemania) comenzando en el primer, segundo o tercer día del ciclo con una dosis entre 75 y 150 unidades internacionales (UI). El crecimiento folicular fue monitoreado mediante ecografía transvaginal y los ajustes de dosis se realizaron hasta que los folículos presentaban un diámetro entre 18 y $20 \mathrm{~mm}$. Para desencadenar la ovulación se utilizaron 5000 IU de gonadotropina coriónica humana urinaria (Gonagam-hCG, Zone Pharma, Baesweiler, Alemania), 6500 UI de hCG recombinante (Ovidrel $®$, Merck, Darmstadt, Alemania) o $0,5 \mathrm{mg}$ de acetato de leuprolida (Lupron ${ }^{\circ}$, Abbott, Chicago, EE.UU.). 
Las muestras de semen se seleccionaron usando el método de swim-up. En todos los casos, la muestra se lavó con All Grad Wash (LifeGlobal, Connecticut, EE.UU.) dos veces por centrifugación y el sedimento se resuspendió en $0,7 \mathrm{~mL}$ con All Grad Wash, finalmente se evaluaron la concentración, la movilidad progresiva y la morfología espermática.

En todos los casos, se realizó la inseminación de 12 a 14 horas después de la inyección de de hCG. Todas las pacientes recibieron $400 \mathrm{mg} /$ día de progesterona micronizada (Utrogestan, Biopas Laboratories, Bogotá, Colombia), después del procedimiento hasta el día de la prueba de embarazo. Las muestras de suero se obtuvieron 14 a 15 días después de la administración de hCG para evaluar sus niveles de $\beta$ hCG.

\section{Análisis Estadístico}

Los datos fueron analizados utilizando el software estadístico Prism 7.0 (GraphPad Software, San Diego, CA) y un valor $p<0,05$ se consideró estadísticamente significativo. Todos los datos están expresados como la media \pm error estándar de la media (SEM).

\section{Resultados}

En el presente estudio, se obtuvieron 71 embarazos de 419 ciclos de IIU, teniendo en total una tasa de embarazo del $16,9 \%$.

La media de la edad masculina y femenina en el momento de la inseminación fue de $37,1 \pm 0,7$ años (media \pm SEM) y $33,41 \pm 0,5$ años, respectivamente. No hubo diferencias en la edad de los hombres y las mujeres, entre las pacientes que obtuvieron un embarazo y aquellas que no lo obtuvieron.

En la - Tabla 1, se comparan los parámetros seminales en los grupos de parejas que no lograron el embarazo frente a las

Tabla 1 Comparación de los parámetros seminales en los grupos de embarazo y no embarazo en muestras frescas y lavadas

\begin{tabular}{|c|c|c|c|}
\hline $\begin{array}{l}\text { Parámetros } \\
\text { seminales }\end{array}$ & $\begin{array}{l}\text { No embarazo } \\
\text { (media } \pm \text { EEM) }\end{array}$ & $\begin{array}{l}\text { Embarazo } \\
\text { (media } \pm \text { EEM) }\end{array}$ & Valor $\mathrm{p}$ \\
\hline \multicolumn{4}{|l|}{ Muestra inicial } \\
\hline $\begin{array}{l}\text { Concentración } \\
\left(10^{6} / \mathrm{mL}\right)\end{array}$ & $73,7 \pm 2,3$ & $81,1 \pm 5,3$ & $>0,05$ \\
\hline Volumen (mL) & $3,5 \pm 0,1$ & $3,7 \pm 0,2$ & $>0,05$ \\
\hline Movilidad (\%) & $40,34 \pm 9,49$ & $40,21 \pm 7,71$ & $>0,05$ \\
\hline Morfología (\%) & $3,7 \pm 0,2$ & $3,5 \pm 0,3$ & $>0,05$ \\
\hline $\begin{array}{l}\text { Número total de } \\
\text { espermatozoides } \\
\text { móviles (TMSC, } 10^{6} \text { ) }\end{array}$ & $104 \pm 4,6$ & $120,2 \pm 11,7$ & $>0,05$ \\
\hline Móviles totales (TM) & $30,8 \pm 1,1$ & $33,2 \pm 2,4$ & $>0,05$ \\
\hline \multicolumn{4}{|l|}{ Muestra procesada } \\
\hline $\begin{array}{l}\text { Concentración } \\
\left(10^{6} / \mathrm{mL}\right)\end{array}$ & $47,9 \pm 1,6$ & $51,5 \pm 3,3$ & $>0,05$ \\
\hline Movilidad (\%) & $81,1 \pm 0,6$ & $84,4 \pm 0,7$ & $>0,05$ \\
\hline Morfología (\%) & $3,5 \pm 0,1$ & $3,6 \pm 0,2$ & $>0,05$ \\
\hline $\begin{array}{l}\text { Movilidad total } \\
\left(\mathrm{TM}, 10^{6}\right)\end{array}$ & $39,82 \pm 1,4$ & $43,7 \pm 2,9$ & $>0,05$ \\
\hline
\end{tabular}

Abreviaciones: EER, erro estándar de la media. que lo lograron. De todas las características espermáticas estudiadas, ninguna mostró diferencias significativas entre los grupos antes y después del procesamiento de la muestra de semen. Sin embargo, las muestras en el grupo de embarazo evidenciaron mejores parámetros seminales, aunque no fueron estadísticamente significativos.

Por otro lado, la movilidad mejoró después del proceso de selección $(p<0,0001)$, la concentración espermática disminuyó $(p<0,0001)$ y la morfología no vario $(p<0,05)$. Esos cambios no tuvieron efecto en las tasas de embarazo clínicas debido a que resultados similares fueron observados en ambos grupos ( - Fig. $\mathbf{1}$ ).

Finalmente, al analizar los parámetros seminales en relación con la edad masculina, este estudio encontró diferencias significativas en las muestras frescas (no separadas), entre los hombres menores (284 pacientes) y los mayores de 40 años (127 pacientes): volumen $(3,6 \pm 0,1 \mathrm{~mL}$ versus $3,2 \pm 0,1 \mathrm{~mL}, p<0,01)$ y en el número total de espermatozoides móviles $\left(114,2 \pm 5,5 \times 10^{6}\right.$ versus $\left.87,2 \pm 6,10,{ }^{6} \quad p<0,01\right)$. Sin embargo, después del procesamiento del semen, no se encontraron diferencias en los parámetros seminales entre los dos grupos.

Es importante aclarar que cuando se eliminaron los parámetros seminales y los resultados de embarazo de los pacientes que repitieron el proceso de IIU, los resultados de los análisis estadísticos fueron los mismos.

\section{Discusión}

La IIU es una técnica de reproducción asistida de baja complejidad con tasas de éxito aceptables para el tratamiento de diferentes alteraciones en las parejas infértiles. $^{10,11}$ Por lo tanto, en el presente trabajo, se evaluaron varios parámetros seminales como predictores de un resultado exitoso después de realizar una IIU. Este estudio intenta evidenciar los efectos de las características espermáticas antes y después de seleccionar los espermatozoides de las muestras de semen con el embarazo después de realizar una IIU.

En el presente estudio no se encontró un parámetro relevante que pueda predecir un embarazo después de la IIU, tanto en muestras frescas como seleccionadas. Esos resultados son similares a los obtenidos por Luco y col., ${ }^{12}$ quienes concluyeron después de realizar 357 inseminaciones que no existe un parámetro seminal predictor del éxito de la IIU. ${ }^{12}$ Un estudio, recientemente publicado por Atasever y col., ${ }^{13}$ también soporta esos resultados en pacientes diagnosticados con infertilidad de origen desconocido e infertilidad masculina leve. ${ }^{13}$

Adicionalmente, Zhao y col., ${ }^{4}$ observaron que la movilidad inicial fue significativamente más alta en pacientes que lograron un embarazo y que la movilidad post selección no fue diferente entre el grupo de pacientes embarazadas y el grupo de pacientes no embarazadas, lo cual es consistente con el presente estudio. Adicionalmente, no encontraron un efecto significativo en el resultado de la IIU relacionado con la concentración espermática inicial, la movilidad y el número total de espermatozoides móviles. ${ }^{4}$ Algunos estudios sugieren 


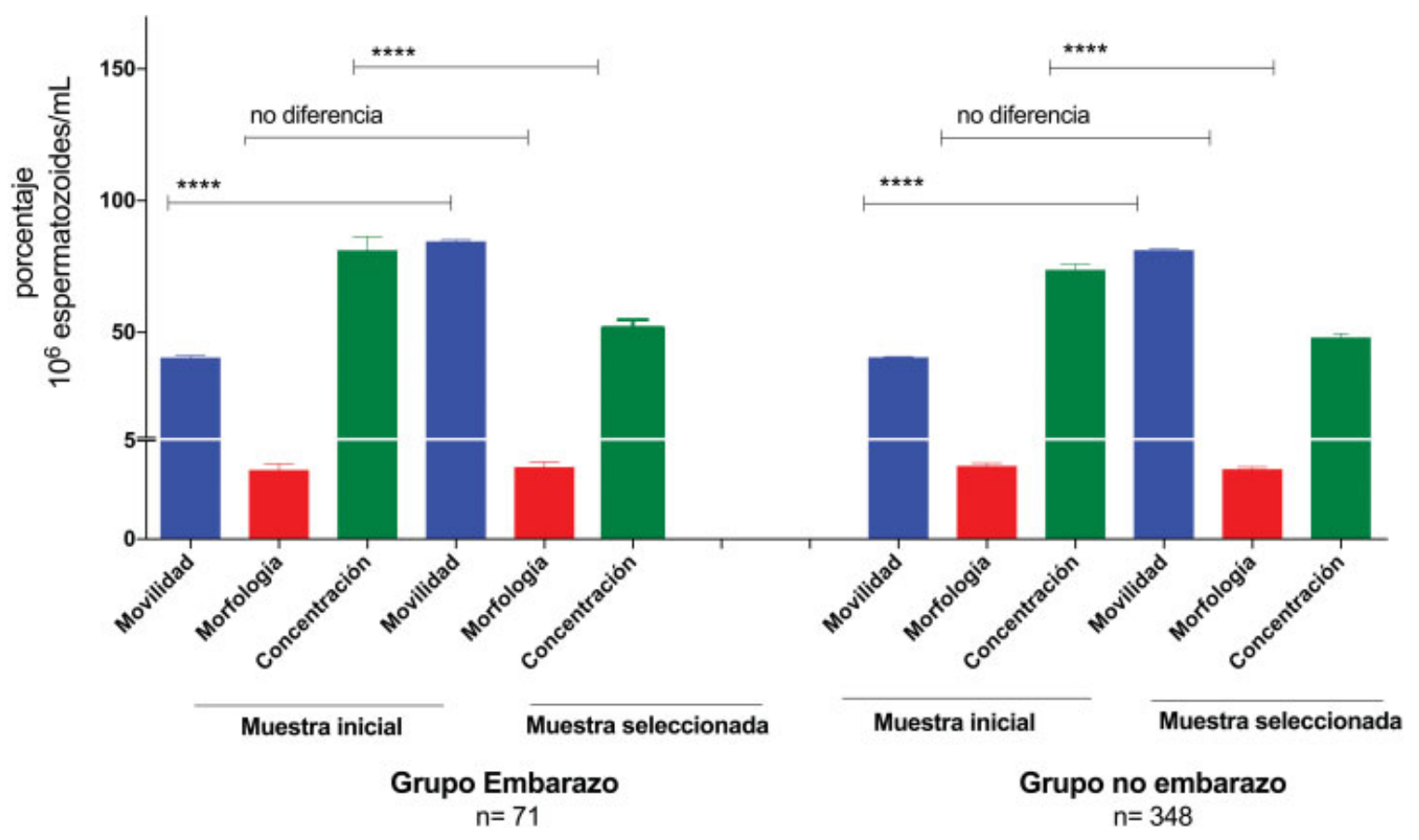

Fig. 1 Parámetros espermáticos pre y post selección en los grupos divididos según el éxito gestacional.

que el conteo total de espermatozoides móviles en fresco debe ser al menos de 5 millones, ${ }^{14,15}$ mientras que otros insisten en que debe ser de al menos 10 millones. ${ }^{16,17}$ De hecho, una revisión sistemática de la literatura realizada por Ombelet y col., ${ }^{15}$ confirma que el número total de espermatozoides móviles en las muestras en fresco presenta una mejor correlación con el éxito de la IIU cuando el rango es entre 5 y 10 millones de espermatozoides $/ \mathrm{mL} ;{ }^{15}$ así, de acuerdo a esos resultados, el número total de espermatozoides móviles es el mejor predictor de embarazo comparado con los parámetros seminales convencionales. ${ }^{18}$

Tjon-Kon-Fat y col., ${ }^{19}$ llegaron a la conclusión que el número total inicial de espermatozoides móviles es el mejor predictor del éxito reproductivo. No obstante, si el número total de espermatozoides móviles es menor a 10 millones de espermatozoides/mL, esos investigadores recomiendan realizar una fertilización in vitro (FIV). ${ }^{19}$

Wang y col., ${ }^{20}$ confirman que si el número total de espermatozoides móviles después de la selección espermática es mayor o igual a 5 millones, se puede considerar como el parámetro principal para lograr un embarazo después de realizar la inseminación, conclusión conseguida después de analizar 379 ciclos de IIU. $^{20}$ Resultados similares fueron obtenidos por otros autores quienes aseguran que ese parámetro es el más relacionado con el embarazo. ${ }^{21,22}$ Sin embargo, mencionan que el éxito del número total de espermatozoides móviles depende de los pacientes de cada clínica y de la metodología para la preparación del semen, ${ }^{21}$ en contraste, otro estudio afirma que el número mínimo de espermatozoides inseminados debe ser de 1 millón. ${ }^{22}$

De acuerdo con la morfología espermática estricta, aunque es un componente esencial del análisis del semen, parece no afectar el hecho de obtener un embarazo. En el presente estudio, no se encontró diferencia estadísticamente significativa entre los grupos (embarazo vs no embarazo) en relación con la morfología de los espermatozoides. Resultados similares han sido obtenidos por otros autores y sugieren que ese parámetro por sí solo no es confiable, debido a la subjetividad inherente y la creciente proporción de hombres diagnosticados erróneamente con morfología anormal, causando una pérdida de su valor predictivo. ${ }^{23-26}$ Así, Shabtaie y col., ${ }^{23}$ recomienda realizar la inseminación a pesar de la morfología anormal considerada por el manual actual de la OMS ( $<4 \%$ de formas normales), ${ }^{9}$ siempre y cuando las muestras seminales presenten movilidad y concentración espermáticas normales. ${ }^{23}$ En contraste, en un estudio prospectivo realizado por Butcher y col., ${ }^{27}$ se encontró en 1243 ciclos de IIU que altos niveles de morfología normal ( $\geq 18 \%$ de formas normales), son un indicador clínico positivo y podría incrementar la probabilidad de embarazo. ${ }^{27}$

Después del procesamiento del semen, se espera que la técnica de selección mejore la calidad de los parámetros seminales. En el presente estudio, la movilidad mejoró, la concentración espermática disminuyó y no hubo variación en el porcentaje de formas normales. Ruiter-Ligeti y col., ${ }^{28}$ encontraron que el procesamiento de la muestra de semen mejora en la mayoría de los parámetros espermáticos con excepción del número total de espermatozoides móviles, el cual es considerado como el parámetro cínicamente más importante. ${ }^{28}$

De otro lado, la influencia de la edad masculina sobre la fertilidad es un área de investigación cada vez más relevante y actualmente se sabe muy poco sobre ese factor en comparación con los bien investigados efectos de la edad femenina. De hecho, en el presente estudio se observó una diferencia significativa en el volumen y en el número total de 
espermatozoides móviles entre hombres menores y mayores de 40 años en las muestras frescas. Sin embargo, en los parámetros seminales después de la selección espermática no hubo diferencias.

Esos datos son respaldados por estudios que muestran una disminución significativa en el volumen, y por lo tanto en el número total de espermatozoides y en el número total de espermatozoides móviles. $^{29-31}$

Aparentemente, a medida que la edad aumenta, se presentan cambios anatómicos y hormonales que podrían resultar en el deterioro de los parámetros seminales. ${ }^{32} \mathrm{Se}$ conoce muy bien que la mayor parte del volumen del semen se origina en las vesículas seminales y se ha reportado que ese volumen disminuye con el avance de la edad. ${ }^{33}$ Eso también está relacionado con una reducción en la producción del fluido andrógeno-estimulante en la próstata y en las vesículas seminales, debido a que los niveles de testosterona disminuyen con la edad. ${ }^{34}$ Así mismo, se producen cambios en la próstata, como la atrofia del músculo liso y una disminución en el contenido de proteínas y agua, que pueden afectar el volumen de semen. ${ }^{35}$ Sin embargo, ninguno de esos estudios pudo demostrar una correlación significativa entre la edad paterna y otras características espermáticas.

Kidd y col., ${ }^{36}$ realizaron un estudio para establecer la asociación entre la edad masculina, la calidad del semen y el estado de la fertilidad. Sus resultados muestran que postergar la paternidad disminuye la calidad del semen en el volumen, la movilidad y morfología de los espermatozoides y, por tanto, la fertilidad, debido a que aumenta el tiempo para conseguir un embarazo. ${ }^{37}$ Otros estudios afirman que la edad paterna se refleja sobre todo en la fertilización, el desarrollo embrionario y la implantación. ${ }^{38-40}$

En el caso de las mujeres, ha sido muy bien estudiado y establecido que a partir de los 35 años de edad la reserva ovárica disminuye considerablemente y por lo tanto la probabilidad de embarazo. Sin embargo, para los hombres no hay punto de corte debido a la falta de estudios. De la Rochebrochard y Thonneau (2003), llevaron a cabo un estudio en el que proponían que a partir de los 40 años de edad, el riesgo de presentar un factor masculino alterado aumenta en comparación con hombres más jóvenes. ${ }^{41}$ En nuestro estudio, encontramos una diferencia significativa en el volumen de semen cuando se utiliza 40 años como un punto de corte.

En conclusión, el presente estudio no encontró ningún parámetro seminal relevante como predictor de éxito de la IIU. Eso puede deberse a que el logro de un embarazo es multifactorial y es necesario tener en cuenta variables como la edad de la mujer, el protocolo de estimulación y la duración de la infertilidad, entre otros. El procesamiento del semen en el laboratorio mejora la movilidad de los espermatozoides y la IIU tiene tasas de embarazo aceptables, por lo que debe considerarse como un tratamiento de primera elección. Por último, aunque la edad masculina tiene un efecto sobre el volumen del semen, no afecta negativamente las tasas de embarazo.

\section{Responsabilidades éticas}

Protección de personas y animales. Los autores declaran que para esta investigación no se han realizado experimentos en seres humanos ni en animales.

Confidencialidad de los datos. Los autores declaran que han seguido los protocolos de su centro de trabajo sobre la publicación de datos de pacientes.

Derecho a la privacidad y consentimiento informado. Los autores han obtenido el consentimiento informado de los pacientes y/o sujetos referidos en el artículo. Este documento obra en poder del autor de correspondencia.

\section{Referencias}

1 Meniru G. Intrauterine insemination. Cambridge Guide to Infertility Management and Assited Reproduction. 1rs ed. Cambridge: Cambridge University Press; 2004

2 Vargas-Hernández VM, Tovar-Rodríguez JM, Acosta-Altamirano G, Moreno-Eutimio MA. Papel de la inseminación intrauterina en la era de la fertilización in vitro. Clin Invest Ginecol Obstet 2014; 41(01):29-34

3 Badawy A, Elnashar A, Eltotongy M. Effect of sperm morphology and number on success of intrauterine insemination. Fertil Steril 2009;91(03):777-781

4 Zhao Y, Vlahos N, Wyncott D, et al. Impact of semen characteristics on the success of intrauterine insemination. J Assist Reprod Genet 2004;21(05):143-148

5 Duran HE, Morshedi M, Kruger T, Oehninger S. Intrauterine insemination: a systematic review on determinants of success. Hum Reprod Update 2002;8(04):373-384

6 Devranoğlu B, Özdamar Ö, Köle E, Eken MK, Bozdağ H, Doğer E. Do younger women with elevated basal follicular stimulating hormone levels undergoing gonadotropin-stimulated intrauterine insemination cycles represent compromised reproductive outcomes? Eur J Obstet Gynecol Reprod Biol 2016;199:141-145

7 Hansen KR, He ALW, Styer AK, et al; Eunice Kennedy Shriver National Institute of Child Health and Human Development Reproductive Medicine Network. Predictors of pregnancy and live-birth in couples with unexplained infertility after ovarian stimulation-intrauterine insemination. Fertil Steril 2016;105 (06):1575-1583.e2

8 Moro F, Tropea A, Scarinci E, et al. Anti-Müllerian hormone concentrations and antral follicle counts for the prediction of pregnancy outcomes after intrauterine insemination. Int $\mathrm{J}$ Gynaecol Obstet 2016;133(01):64-68

9 WHO. WHO laboratory manual for the Examination and processing of human semen. fifth edit. WHO Library; 2010

10 Erdem M, Abay S, Erdem A, et al. Recombinant FSH increases live birth rates as compared to clomiphene citrate in intrauterine insemination cycles in couples with subfertility: a prospective randomized study. Eur J Obstet Gynecol Reprod Biol 2015;189:33-37

11 Yumusak OH, Kahyaoglu S, Pekcan MK, Isci E, Cinar M, Tasci Y. Does intrauterine insemination timing matter for achieving pregnancy during ovulation induction using gonadotropins? A retrospective cohort study. J Chin Med Assoc 2017;80:366-370

12 Luco SM, Agbo C, Behr B, Dahan MH. The evaluation of pre and post processing semen analysis parameters at the time of intrauterine insemination in couples diagnosed with male factor infertility and pregnancy rates based on stimulation agent. A retrospective cohort study. Eur J Obstet Gynecol Reprod Biol 2014;179:159-162

13 Atasever M, Kalem MN, Hatırnaz S, Hatırnaz E, Kalem Z, Kalaylıoğlu Z. Factors affecting clinical pregnancy rates after IUI for the treatment of unexplained infertility and mild male subfertility. J Turk Ger Gynecol Assoc 2016;17(03):134-138 
14 Merviel P, Heraud MH, Grenier N, Lourdel E, Sanguinet P, Copin H. Predictive factors for pregnancy after intrauterine insemination (IUI): an analysis of 1038 cycles and a review of the literature. Fertil Steril 2010;93(01):79-88

15 Ombelet W, Dhont N, Thijssen A, Bosmans E, Kruger T. Semen quality and prediction of IUI success in male subfertility: a systematic review. Reprod Biomed Online 2014;28(03):300-309

16 Miller DC, Hollenbeck BK, Smith GD, et al. Processed total motile sperm count correlates with pregnancy outcome after intrauterine insemination. Urology 2002;60(03):497-501

17 Dorjpurev U, Kuwahara A, Yano Y, et al. Effect of semen characteristics on pregnancy rate following intrauterine insemination. J Med Invest 2011;58(1-2):127-133

18 Papillon-Smith J, Baker SE, Agbo C, Dahan MH. Pregnancy rates with intrauterine insemination: comparing 1999 and 2010 World Health Organization semen analysis norms. Reprod Biomed Online 2015;30(04):392-400

19 Tjon-Kon-Fat RI, Tajik P, Custers IM, et al. Can we identify subfertile couples that benefit from immediate in vitro fertilisation over intrauterine insemination? Eur J Obstet Gynecol Reprod Biol 2016;202:36-40

20 Wang L, Sun N, Lu X, et al. Effect of Timing and Number of Intrauterine Insemination (IUI) on the Cycle Clinical Outcome. J Reprod Contracept 2014;25(04):219-226

21 van Weert JM, Repping S, Van Voorhis BJ, van der Veen F, Bossuyt PMM, Mol BWJ. Performance of the postwash total motile sperm count as a predictor of pregnancy at the time of intrauterine insemination: a meta-analysis. Fertil Steril 2004;82(03): 612-620

22 Dinelli L, Courbière B, Achard V, et al. Prognosis factors of pregnancy after intrauterine insemination with the husband's sperm: conclusions of an analysis of 2,019 cycles. Fertil Steril 2014;101(04):994-1000

23 Shabtaie SA, Gerkowicz SA, Kohn TP, Ramasamy R. Role of Abnormal Sperm Morphology in Predicting Pregnancy Outcomes. Curr Urol Rep 2016;17(09):67

24 Lemmens L, Kos S, Beijer C, et al; Semen Section of the Dutch Foundation for Quality Assessment in Medical Laboratories. Predictive value of sperm morphology and progressively motile sperm count for pregnancy outcomes in intrauterine insemination. Fertil Steril 2016;105(06):1462-1468

25 Erdem M, Erdem A, Mutlu MF, et al. The impact of sperm morphology on the outcome of intrauterine insemination cycles with gonadotropins in unexplained and male subfertility. Eur J Obstet Gynecol Reprod Biol 2016;197:120-124

26 Deveneau NE, Sinno O, Krause M, et al. Impact of sperm morphology on the likelihood of pregnancy after intrauterine insemination. Fertil Steril 2014;102(06):1584-90.e2
27 Butcher MJ, Janoo J, Broce M, Seybold DJ, Gantt P, Randall G. Use of Sperm Parameters to Predict Clinical Pregnancy with Intrauterine Insemination. J Reprod Med 2016;61(5-6):263-269

28 J. Ruiter-Ligeti CA. Dahan M. The impact of semen processing on sperm quality and pregnancy rates with intrauterine inseminations. Fertil Steril 2015;104(03):e246

29 Bellver J, Garrido N, Remohí J, Pellicer A, Meseguer M. Influence of paternal age on assisted reproduction outcome. Reprod Biomed Online 2008;17(05):595-604

30 Frattarelli JL, Miller KA, Miller BT, Elkind-Hirsch K, Scott RT Jr. Male age negatively impacts embryo development and reproductive outcome in donor oocyte assisted reproductive technology cycles. Fertil Steril 2008;90(01):97-103

31 Luna M, Finkler E, Barritt J, et al. Paternal age and assisted reproductive technology outcome in ovum recipients. Fertil Steril 2009;92(05):1772-1775

32 Dain L, Auslander R, Dirnfeld M. The effect of paternal age on assisted reproduction outcome. Fertil Steril 2011;95(01):1-8

33 Hayakawa T, Naya Y, Kojima M. Significant changes in volume of seminal vesicles as determined by transrectal sonography in relation to age and benign prostatic hyperplasia. Tohoku J Exp Med 1998;186(03):193-204

34 Goldman N, Montgomery M. Fecundability and husband's age. Soc Biol 1989;36(3-4):146-166

35 Harman S. Clinical aspects of aging of the male reproductive system. In: Schneider EL, editor. The aging reproductive system. New York: Raven Press; 1978

36 Kidd SA, Eskenazi B, Wyrobek AJ. Effects of male age on semen quality and fertility: a review of the literature. Fertil Steril 2001; 75(02):237-248

37 Hassan MAM, Killick SR. Effect of male age on fertility: evidence for the decline in male fertility with increasing age. Fertil Steril 2003;79(Suppl 3):1520-1527

38 Klonoff-Cohen HS, Natarajan L. The effect of advancing paternal age on pregnancy and live birth rates in couples undergoing in vitro fertilization or gamete intrafallopian transfer. Am J Obstet Gynecol 2004;191(02):507-514

39 Duran EH, Dowling-Lacey D, Bocca S, Stadtmauer L, Oehninger S. Impact of male age on the outcome of assisted reproductive technology cycles using donor oocytes. Reprod Biomed Online 2010;20(06):848-856

40 Raberi A, Rozis G, Alfarawati S, et al. A comprehensive study into the effects of advancing male age on semen parameters, sperm genetic integrity and the outcome of assisted reproductive treatments. Fertil Steril 2016;106(03):e304

41 de La Rochebrochard E, Thonneau P. Paternal age $>$ or $=40$ years: an important risk factor for infertility. Am J Obstet Gynecol 2003; 189(04):901-905 